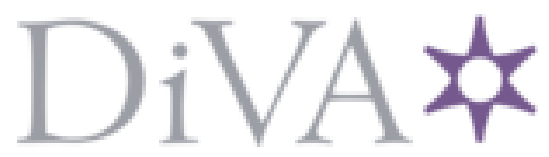

http://www.diva-portal.org

This is the published version of a paper published in Physical Review B. Condensed Matter and Materials Physics.

Citation for the original published paper (version of record):

Jääskeläinen, M., Zulicke, U. (2010)

Anomalous spin-related quantum phase in mesoscopic hole rings.

Physical Review B. Condensed Matter and Materials Physics, 81(15): 155326

http://dx.doi.org/10.1103/PhysRevB.81.155326

Access to the published version may require subscription.

N.B. When citing this work, cite the original published paper.

Permanent link to this version:

http://urn.kb.se/resolve?urn=urn:nbn:se:du-11201 


\title{
Anomalous spin-related quantum phase in mesoscopic hole rings
}

\author{
M. Jääskeläinen \\ Institute of Fundamental Sciences and MacDiarmid Institute for Advanced Materials and Nanotechnology, Massey University, \\ Manawatu Campus, Private Bag 11 222, Palmerston North 4442, New Zealand \\ U. Zülicke \\ Institute of Fundamental Sciences and MacDiarmid Institute for Advanced Materials and Nanotechnology, Massey University, \\ Manawatu Campus, Private Bag 11 222, Palmerston North 4442, New Zealand \\ and Centre for Theoretical Chemistry and Physics, Massey University, Albany Campus, \\ Private Bag 102904, North Shore MSC, Auckland 0745, New Zealand
}

(Received 21 January 2010; revised manuscript received 26 March 2010; published 29 April 2010)

\begin{abstract}
We have obtained numerically exact results for the spin-related geometric quantum phases that arise in p-type semiconductor ring structures. The interplay between gate-controllable (Rashba) spin splitting and quantum-confinement-induced mixing between hole-spin states causes a much higher sensitivity of magnetoconductance oscillations to external parameters than previously expected. Our results imply a much-enhanced functionality of hole-ring spin-interference devices and shed new light on recent experimental findings.
\end{abstract}

DOI: 10.1103/PhysRevB.81.155326

PACS number(s): 85.35.Ds, 03.65.Vf, 71.70.Ej, 73.23.Ad

\section{INTRODUCTION, MOTIVATION AND SUMMARY}

Quantum-interference effects dominate electric transport through conductors that are mesoscopic, i.e., have a smaller size than the decoherence length set by inelastic interactions of charge carriers with other degrees of freedom (e.g., phonons, disorder). ${ }^{1}$ In particular, mesoscopic ring structures exhibit magnetoconductance oscillations ${ }^{2}$ that reveal geometric quantum [Berry, ${ }^{3}$ Aharonov-Anandan (AA) (Refs. 4 and 5)] phases acquired by charge carriers propagating quantum coherently through a multiply connected geometry. Coupling of orbital motion to the spin of charge carriers affects the quantum interference and geometric phases manifested in charge transport through rings. ${ }^{6}$ Such spin-dependent electronic interference effects could form the operational basis for novel transistor devices ${ }^{7}$ and quantum logic gates. ${ }^{8}$

Strong experimental efforts have been undertaken to identify and measure spin-related geometric phases in magnetotransport through arrays of mesoscopic rings, ${ }^{9,10}$ singlering structures, ${ }^{11-15}$ and antidot superlattices. ${ }^{16}$ Many recent experiments were performed in p-type semiconductor structures ${ }^{11,14-16}$ because charge carriers from the valence band (holes) are expected to be subject to much larger momentum-dependent spin splittings than conduction-band electrons. ${ }^{17}$ In contrast, many theoretical works have considered spin-dependent interference in $n$-type semiconductor rings ${ }^{18-23}$ while the electronic properties of $p$-type rings remain largely unexplored. As conduction-band electrons and valence-band holes are not merely distinguished by the sign of their charge but are known to exhibit very different spin properties, especially in quantum-confined structures, ${ }^{17}$ a careful analysis of tunable spin-related quantum phases in p-type mesoscopic rings is needed.

Recent calculations ${ }^{24-26}$ of the magnetoconductance in hole rings adopted a purely heavy-hole $(\mathrm{HH})$ model where only the valence-band states with spin projection $\pm 3 / 2$ (heavy holes) and their effective Rashba-type spin splitting are taken into account. It is tempting to follow such a route because the highest quasi-two-dimensional (2D) valence subband is mostly of $\mathrm{HH}$ character for typical hole sheet densities, ${ }^{17}$ and the $\mathrm{HH}$ model bears resemblance to the one that applies to conduction-band electrons. However, such an approach neglects the hole-spin mixing induced by quantum confinement in ring structures. Here we report results of a theoretical study that fully accounts for spin splitting and spin mixing in the valence band. Interestingly, we find a synergistic relation between gate-tunable Rashba spin splitting, which arises from the structural inversion asymmetry (SIA) in the 2D semiconductor heterostructure, and the holespin mixing due to the ring confinement. This is illustrated in Fig. 1 where the dependence of magnetoconductance oscillations on the Fermi energy in the hole-ring structure is shown. ${ }^{27}$ The more complete theory underlying our calculation predicts a much more frequent change between maxima and minima of the magnetoconductance as a function of the Fermi energy than is found for the same ring geometry and materials parameters within the $\mathrm{HH}$ model. Hence, an analysis of experimental databased on the latter would have to assume an unrealistically large SIA in the measured sample. Conversely, our results suggest that moderate changes in hole density and/or SIA, routinely achieved using gate voltages, will be sufficient to operate a spin-interference-based nanoelectronic device.

This paper is organized as follows. Section II presents our theoretical model for mesoscopic hole rings. Zero-field spin splitting of holes due to SIA is discussed in Sec. III. Section IV focuses on the spin-related Aharonov-Anandan phase and how it is revealed in magnetoconductance oscillations. The frequently used ${ }^{24-26}$ heavy-hole model for $p$-type mesoscopic rings is introduced in Sec. V and results obtained using it are compared with those found within our more complete theory. The penultimate Sec. VI presents an interpretation of recent experiments in light of our new results, discussing also possible effects of spin splitting due to bulk inversion asymmetry (BIA). Our conclusions are given in Sec. VII. Some relevant mathematical derivations are given in the Appendix. 


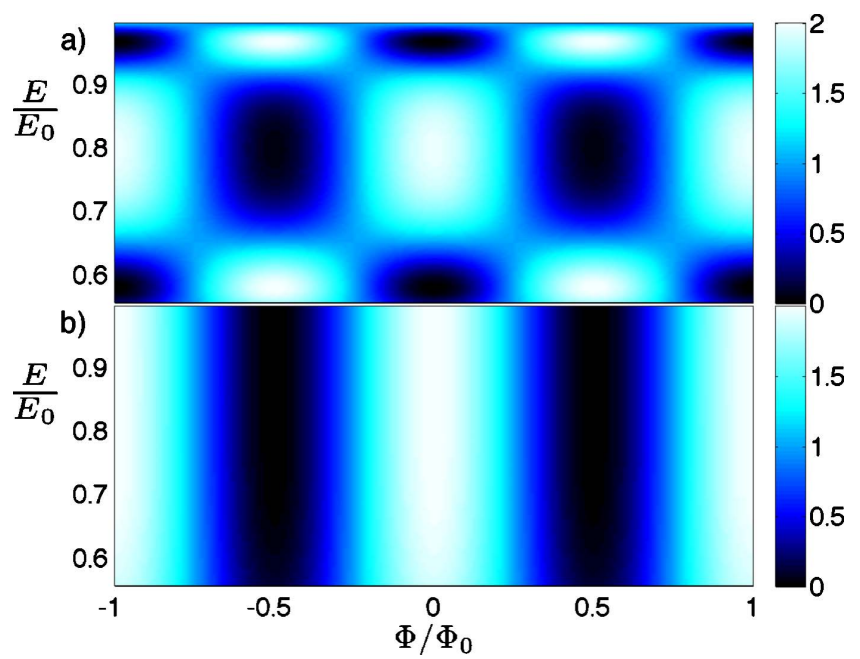

FIG. 1. (Color online) Dependence of hole-ring magnetoconductance oscillations on the Fermi energy $E$. The latter is linearly related to the hole sheet density in the semiconductor heterostructure and can be controlled by gate voltages. $\Phi$ denotes the magnetic flux penetrating the ring and $\Phi_{0}$ is the flux quantum. Panel (a) shows results from calculations based on our more complete theory, whereas use of the simplified heavy-hole model for the same ring device yields panel (b). See text for more details. The range of energies shown corresponds to the situation where only the lowest ring subband is occupied.

\section{MODEL FOR A MESOSCOPIC HOLE RING}

Our calculations are based on the $4 \times 4$ Luttinger model for the uppermost valence band in typical semiconductors, ${ }^{28}$ which takes both the heavy-hole and the light-hole states into account. For simplicity, we neglect band warping due to the cubic crystal symmetry. The ring confinement is assumed to be due to a quantum-well potential in $z$ direction (width $d$ ) and a singular-oscillator potential $V_{\perp}(r)=m_{0} \omega^{2}$ $\left(r-\left[R^{2} / r\right]\right)^{2} / 2$ for the radial coordinate $r$ in the $x y$ plane. Here $R$ is the effective ring radius, and the oscillator potential defines a length scale $\ell_{\omega}=\sqrt{\sqrt{\gamma_{1}} \hbar /\left(m_{0} \omega\right)}$ that is a measure of the in-plane ring width. Only the lowest 2D quantumwell bound state is taken into account, hence our theory applies in the (typically realistic) case $d \ll \ell_{\omega}$. The energy splitting between 2D heavy-hole and light-hole subband edges is accounted for by the Hamiltonian (we use the hole picture for the valence band, counting energies as positive from the bulk valence-band edge)

$$
H_{\mathrm{qw}}=\left(1-2 \bar{\gamma}\left[\hat{J}_{z}^{2}-\frac{5}{4}\right]\right) E_{0},
$$

where $E_{0}=\pi^{2} \hbar^{2} \gamma_{1} /\left(2 m_{0} d^{2}\right), \hat{J}_{z}$ is the operator for the spin$3 / 2$ angular-momentum component perpendicular to the ring plane, and $\bar{\gamma}=\left(2 \gamma_{2}+3 \gamma_{3}\right) /\left(5 \gamma_{1}\right)$ in terms of Luttinger parameters. ${ }^{28}$ The in-plane hole motion is governed by

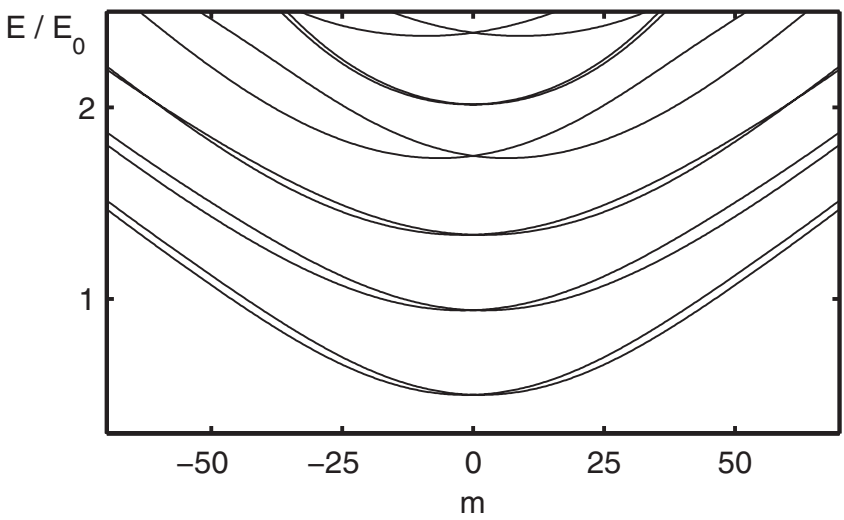

FIG. 2. Hole-ring subbands in a quantum well without SIA. Parameters: in-plane aspect ratio (radius/width) $\sqrt{\lambda_{R}}=20, \lambda_{d}=0.5$, and $\bar{\gamma}=0.37$ (value applies to GaAs). $m$ is the eigenvalue of total angular-momentum component $\hat{M}_{z}=\hat{L}_{z}+\hat{J}_{z}$ perpendicular to the ring. $E_{0}=\pi^{2} \hbar^{2} \gamma_{1} /\left(2 m_{0} d^{2}\right)$ is the energy scale set by size quantization in the $2 \mathrm{D}$ quantum well from which the ring is fabricated.

$$
\begin{aligned}
H_{\mathrm{rg}}= & \frac{\lambda_{d}}{4}\left\{\left(1+\bar{\gamma}\left[\hat{J}_{z}^{2}-\frac{5}{4}\right]\right) \ell_{\omega}^{2} \hat{k}_{\perp}^{2}-\bar{\gamma} \ell_{\omega}^{2}\left(\hat{k}_{-}^{2} \hat{J}_{+}^{2}+\hat{k}_{+}^{2} \hat{J}_{-}^{2}\right)\right. \\
& \left.+\left(\frac{r}{\ell_{\omega}}-\lambda_{R} \frac{\ell_{\omega}}{r}\right)^{2}\right\} E_{0}
\end{aligned}
$$

with $\lambda_{d}=\left(2 d /\left[\pi \ell_{\omega}\right]\right)^{2}$ and $\lambda_{R}=\left(R / \ell_{\omega}\right)^{2}$. Here $\hat{\mathbf{k}}_{\perp}=\left(\hat{k}_{x}, \hat{k}_{y}\right)$ is the in-plane hole wave vector, $\hat{k}_{ \pm}=\hat{k}_{x} \pm i \hat{k}_{y}$ and $\hat{J}_{ \pm}$ $=\left(\hat{J}_{x} \pm i \hat{J}_{y}\right) / \sqrt{2}$.

The hole-ring Hamiltonian $H_{\mathrm{qw}}+H_{\mathrm{rg}}$ commutes with $\hat{M}_{z}$ $=\hat{L}_{z}+\hat{J}_{z}$, where $\hat{L}_{z}=x \hat{k}_{y}-y \hat{k}_{x}$. The eigenvalues $m$ of $\hat{M}_{z}$ can thus be used to label states within the quasi-one-dimensional (1D) ring subbands. ${ }^{29}$ Adopting polar coordinates $r$ and $\varphi$ for the in-plane motion and making the Ansatz

$$
\langle r, \varphi \mid \psi\rangle=e^{i\left(m-\hat{J}_{z}\right) \varphi} \chi_{m}(r)
$$

for the four-spinor hole wave function generates a purely radial Schrödinger equation that we solve numerically using a pseudospectral method ${ }^{30,31}$ tailored to our needs. Figure 2 shows a representative result for ring subbands $E_{s}^{(n)}(m)$, where $s= \pm 1$ distinguishes spin-split dispersions with eigenvalues related via $E_{s}^{(n)}(m)=E_{-s}^{(n)}(-m)$, and $n=0,1, \ldots$ labels the doublets starting with the lowest-lying one.

\section{EFFECTS OF SIA SPIN SPLITTING}

To investigate spin-related geometric phases in hole rings, we include the dominant SIA contribution to the bulk-hole Hamiltonian, ${ }^{17}$ which is given by $\mathcal{H}_{\mathrm{SIA}}^{\text {(bulk) }}=r_{41}^{8 v 8 v}(\hat{\mathbf{k}} \times \mathcal{E}) \cdot \hat{\mathbf{J}}$. In our case of interest, the SIA electric field $\mathcal{E}$ has a $z$ component $\mathcal{E}_{z}$ determined by the $2 \mathrm{D}$ quantum-well confinement. In addition, the radial in-plane (ring) confinement induces an SIA spin splitting. We find $\mathcal{H}_{\mathrm{SIA}}^{(\mathrm{rg})}=H_{\mathrm{SIA}}^{(\mathrm{rg}, \mathrm{qw})}+H_{\mathrm{SIA}}^{(\mathrm{rg}, \mathrm{rg})}$ with

$$
H_{\mathrm{SIA}}^{(\mathrm{rg}, \mathrm{qw})}=\frac{V_{\mathrm{SIA}}}{V_{41}} \sqrt{\frac{\lambda_{d}}{2}} \ell_{\omega} i\left(\hat{k}_{+} \hat{J}_{-}-\hat{k}_{-} \hat{J}_{+}\right) E_{0},
$$




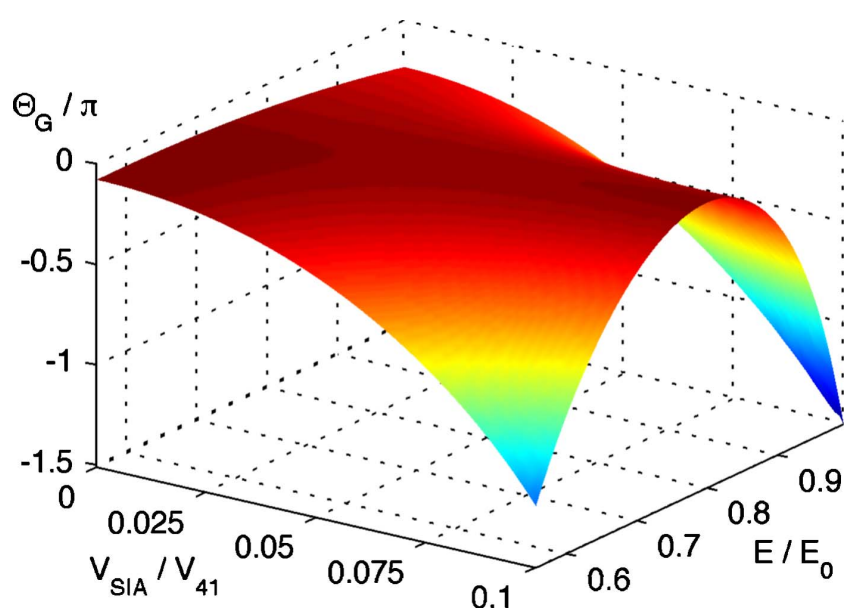

FIG. 3. (Color online) Spin-related quantum (AharonovAnandan) phase for holes in a mesoscopic ring, plotted as a function of hole energy $E$ and the voltage $V_{\text {SIA }}$ associated with SIA in the semiconductor heterostructure from which the ring is fabricated. Other parameters are the same as in Fig. 2. The energy range shown corresponds to the situation where only the lowest ring subband is populated. The band-structure parameter $V_{41}$ is $11.44 \mathrm{~V}$ in GaAs.

$$
H_{\mathrm{SIA}}^{(\mathrm{rg}, \mathrm{rg})}=\lambda_{41} \frac{\lambda_{d}}{2}\left[1-\lambda_{R}^{2}\left(\frac{\ell_{\omega}}{r}\right)^{4}\right] \hat{L}_{z} \hat{J}_{z} E_{0} .
$$

The voltage $V_{\text {SIA }}=\mathcal{E}_{z} d$ is a measure for SIA in the quantum well and $V_{41}=\pi \gamma_{1} \hbar^{2} /\left(m_{0}\left|r_{41}^{8 v 8 v}\right|\right)$ is a materials parameter (11.44 $\mathrm{V}$ in GaAs). $\lambda_{41}=\left|r_{41}^{8 v 8 v}\right| /\left(e \ell_{\omega}^{2}\right)$ is typically very small unless the ring becomes narrow on the scale $\sqrt{\left|r_{41}^{8 v 8 v}\right| / e}$, which is of order angstrom. We checked that matrix elements of Eq. (4b) are negligible for typical hole-ring device parameters. Thus, SIA splitting due to the in-plane ring confinement can be disregarded.

The hole-ring Hamiltonian including the SIA terms still commutes with $\hat{M}_{z}$. Using the same procedure as outlined in Sec. II, we obtain the ring-subband dispersions including the SIA term $H_{\mathrm{SIA}}^{(\mathrm{rg}, \mathrm{qw})}$.

\section{AHARONOV-ANANDAN PHASE AND MAGNETOCONDUCTANCE OSCILLATIONS}

Knowledge of the ring-subband dispersions makes it possible $7,18,19,24,29$ to extract the AA phase ${ }^{4}$ for holes traversing the ring at a particular energy $E$ (generally, the Fermi energy of holes in the 2D semiconductor heterostructure). In the following, we focus entirely on the energy range where only the lowest spin-split ring subband is relevant, but our results can easily be generalized. In terms of the two Fermi angular momenta $m_{ \pm}(E)$ defined by $E=E_{s}^{(0)}\left( \pm s m_{ \pm}\right)$, the AA phase is given by (see the Appendix for details of the derivation)

$$
\Theta_{\mathrm{G}}=\pi\left(m_{+}-m_{-}-3\right) .
$$

The dependence of this spin-related geometric phase on the Fermi energy $E$ and SIA strength $V_{\text {SIA }}$ is shown in Fig. 3 for a set of typical hole-ring parameters.

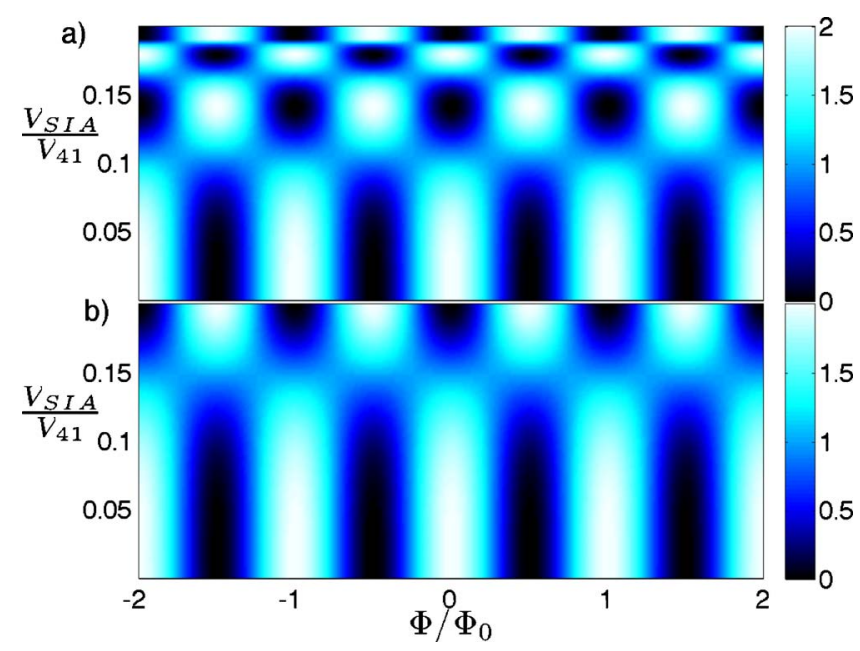

FIG. 4. (Color online) Magnetoconductance oscillations for a mesoscopic hole ring attached to ideal leads, shown as a function of the voltage $V_{\text {SIA }}$ associated with SIA in the semiconductor heterostructure. Panel (a) is obtained from our more complete theory, whereas panel (b) results from application of the $\mathrm{HH}$ model to the same ring device. The band-structure parameter $V_{41}$ is $11.44 \mathrm{~V}$ in GaAs. In the calculation, the Fermi level was assumed to be at $0.6 E_{0}$, and the visibility $A$ was set to 1 . Other parameters are as for Fig. 2.

At low-enough temperatures, the electric conductance $G$ of a mesoscopic ring attached to ideal leads ${ }^{32}$ exhibits a quantum-interference contribution that makes it possible to measure the Aharonov-Anandan phase. Quite generally, it is given by the expression (see the Appendix for more details)

$$
G=G_{0}\left[1+A \cos \left(2 \pi \frac{\Phi}{\Phi_{0}}\right) \cos \Theta_{\mathrm{G}}\right],
$$

where $A \leq 1$ measures the visibility of quantum interference in the ring. The first cosine term contains the magnetic flux $\Phi$ penetrating the ring's area, measured in units of the flux quantum $\Phi_{0}=2 \pi \hbar / e$. It gives rise to magnetoconductance oscillations $^{33}$ that are the electric analog of the AharonovBohm effect. ${ }^{34}$ The modulation of the magnetoconductance as a function of SIA strength achieved, e.g., by external gate voltages, reveals the presence of the spin-related quantum phase $\Theta_{\mathrm{G}}$. This is illustrated in Fig. 4(a).

Experimentally, a change in the strength of SIA while keeping all other parameters (in particular, the hole density) constant can be achieved by simultaneously applied front and back-gate voltages. ${ }^{35}$ However, in the majority of samples, ${ }^{10,11,13,15}$ only a single (front or back) gate is available. In such a situation, both SIA and the density of charge carriers in the semiconductor heterostructure are changed by a gate voltage. For holes, changing the density (i.e., the Fermi energy) has a profound effect on the spin-related quantum phase. This can be inferred from the strong dependence of $\Theta_{\mathrm{G}}$ on $E$ for constant $V_{\mathrm{SIA}}$ seen in Fig. 3. The modulation of magnetoconductance oscillations when changing only the hole density (keeping $V_{\text {SIA }}=0.1 V_{41}$ constant, and with $A=1$ ) is illustrated in Fig. 1(a). Up to a constant shift, $E$ is directly 
proportional to the $2 \mathrm{D}$ sheet density of holes in the heterostructure.

\section{COMPARISON WITH THE HEAVY-HOLE MODEL}

The necessity to fully account for valence-band mixing in hole rings can be illustrated by a direct comparison with the simpler $\mathrm{HH}$ model. The latter results from a perturbative (Löwdin-partitioning) treatment ${ }^{17}$ of valence-band mixing and SIA splitting for the lowest 2D HH subband, neglecting further spin splitting and mixing due to the in-plane confinement. The Hamiltonian of the $\mathrm{HH}$-model ring is $H_{\mathrm{qw}}^{(\mathrm{HH})}$ $+H_{\mathrm{rg}}^{(\mathrm{HH})}+H_{\mathrm{SIA}}^{(\mathrm{HH})}$ with

$$
\begin{gathered}
H_{\mathrm{qw}}^{(\mathrm{HH})}=(1-2 \bar{\gamma}) E_{0}, \\
H_{\mathrm{rg}}^{\mathrm{HH})}=\frac{\lambda_{d}}{4}\left\{(1+\bar{\gamma}) \ell_{\omega}^{2} \hat{k}_{\perp}^{2}+\left(\frac{r}{\ell_{\omega}}-\lambda_{R} \frac{\ell_{\omega}}{r}\right)^{2}\right\} E_{0}, \\
H_{\mathrm{SIA}}^{(\mathrm{HH})}=\frac{V_{\mathrm{SIA}}}{V_{41}}\left(\frac{\lambda_{d}}{8}\right)^{3 / 2} \ell_{\omega}^{3} i\left(\hat{k}_{+}^{3} \hat{J}_{-}^{3}-\hat{k}_{-}^{3} \hat{J}_{+}^{3}\right) E_{0}, \\
\equiv \frac{3}{4} \frac{V_{\mathrm{SIA}}}{V_{41}}\left(\frac{\lambda_{d}}{4}\right)^{3 / 2} \ell_{\omega}^{3} i\left(\hat{k}_{+}^{3} \hat{\sigma}_{-}-\hat{k}_{-}^{3} \hat{\sigma}_{+}\right) E_{0} .
\end{gathered}
$$

The second line defining $H_{\mathrm{SIA}}^{(\mathrm{HH})}$ applies when the $\mathrm{HH}\left(J_{z}\right.$ $= \pm 3 / 2)$ amplitudes are treated as an effective spin-1/2 degree of freedom; this is the way SIA spin splitting for heavy holes is usually written. ${ }^{17}$ No coupling to light-hole amplitudes is present in the $\mathrm{HH}$ model, even after the in-plane ring confinement is introduced.

Using the same numerical method as for the full spin-3/2 Luttinger theory of hole rings outlined above, we find the subbands of the HH-model ring and the spin-related quantum phase associated with the lowest one. Its dependence on both energy $E$ and strength of SIA turns out to be much weaker than in the more complete theory. For the energy dependence of magnetoconductance oscillations, this is illustrated in Fig. 1 for a ring with $V_{\text {SIA }}=0.1 V_{41}$ and all other parameters as in Fig. 2. A similar result is obtained when energy $E$ is fixed and $V_{\text {SIA }}$ is varied, see Fig. 4. The different behavior exhibited by the full Luttinger model as compared with the $\mathrm{HH}$ model arises from HH-LH mixing induced by the in-plane ring confinement. Hence, differences in quantitative predictions from the two models scale with $\lambda_{d}$ and thus vanish in the $2 \mathrm{D}$ limit.

\section{APPLICATION TO REAL HOLE-RING SAMPLES}

Our theory enables a more detailed quantitative interpretation of experimental results. Applied gate voltages have been observed to shift magnetoconductance oscillations. ${ }^{13,15,36}$ Comparison with Shubnikov-de Haas data measured in the unstructured 2D HH system enabled experimentalists to quantify the change in SIA strength required for a $\pi$-phase shift. The $\mathrm{HH}$ model predicts $\Delta V_{\text {SIA }}$ $=V_{41} /\left(n_{\mathrm{F}}^{2} \lambda_{d} \lambda_{R}\right)^{1 / 2}$ for a ring with $n_{\mathrm{F}}$ occupied 1D subbands. ${ }^{24,25}$ Interestingly, the experiment reported in Ref. 15 observed an order-of-magnitude discrepancy between the
TABLE I. Phase shift of magnetoconductance oscillations induced by varying $V_{\text {SIA }} / V_{41}$ between 0.527 and 0.551 , as measured for a 1D GaAs hole ring (Ref. 15) and calculated using the full Luttinger model and the simpler $\mathrm{HH}$ model, respectively. Parameters used in the calculations are $\bar{\gamma}=0.37, \lambda_{d}=1.0, \lambda_{R}=28$, and $E$ $=1.1 E_{0}$ (Luttinger model), $n_{\mathrm{F}}=1$ (HH model). In general, $E$ can be determined from the $2 \mathrm{D}$ hole sheet density and details of the sample's quantum-well confinement.

\begin{tabular}{lcc}
\hline \hline Experiment & Luttinger model & HH model \\
\hline$\pi$ & $0.25 \pi$ & $0.13 \pi$ \\
\hline \hline
\end{tabular}

measured value and that expected from application of the $\mathrm{HH}$ model. As the comparison given in Table I shows, taking into account the enhancement due to $\mathrm{HH}-\mathrm{LH}$ mixing within the full Luttinger model markedly reduces this discrepancy. We suspect that even better agreement could be reached if (a) more details about the ring structure were known, thus facilitating a more realistic modeling of the quantum-well and in-plane confinement potentials and (b) the effect of bandwarping corrections were included. Finally, typical ring devices are fabricated in semiconductors whose unit cell lacks inversion symmetry and, thus, are subject to an additional spin splitting due to BIA. We will briefly discuss BIA effects before concluding.

The most important BIA spin-splitting term in the bulkhole Hamiltonian is ${ }^{17} \mathcal{H}_{\mathrm{BIA}}^{(\text {bulk) }}=b_{41}^{8 v 8 v}\left(\left\{k_{x}, k_{y}^{2}-k_{z}^{2}\right\} J_{x}+\right.$ c.p. $)$. Introducing the $2 \mathrm{D}$ quantum-well confinement by replacing $\hat{k}_{z} \rightarrow\left\langle\hat{k}_{z}\right\rangle \equiv 0$ and $\hat{k}_{z}^{2} \rightarrow\left\langle\hat{k}_{z}^{2}\right\rangle \equiv \pi^{2} / d^{2}$ yields the BIA contribution to the model-ring Hamiltonian as $H_{\mathrm{BIA}}^{(\mathrm{rg})}=H_{\mathrm{BIA}}^{(\mathrm{rg}, \mathrm{qw})}$ $+H_{\mathrm{BIA}}^{\mathrm{rg}, \mathrm{rg})}$, where

$$
\begin{gathered}
H_{\mathrm{BIA}}^{(\mathrm{rg}, \mathrm{qw})}=\frac{\ell_{\mathrm{BIA}}}{d} \sqrt{\frac{\lambda_{d}}{2}} \ell_{\omega}\left(\hat{k}_{+} \hat{J}_{+}+\hat{k}_{-} \hat{J}_{-}\right), \\
H_{\mathrm{BIA}}^{(\mathrm{rg}, \mathrm{rg})}=\frac{\ell_{\mathrm{BIA}}}{d}\left(\frac{\lambda_{d}}{8}\right)^{3 / 2} \ell_{\omega}^{3}\left(\hat{k}_{+}^{2}-\hat{k}_{-}^{2}\right)\left(\hat{k}_{+} \hat{J}_{-}-\hat{k}_{-} \hat{J}_{+}\right) .
\end{gathered}
$$

The length scale $\ell_{\mathrm{BIA}}=\pi m_{0}\left|b_{41}^{8 v 8 v}\right| /\left(\gamma_{1} \hbar^{2}\right) \equiv 4.83 \AA$ in GaAs. As typical quantum-well widths are on the order of $10 \mathrm{~nm}$, we have $\ell_{\mathrm{BIA}} / d \sim 0.05$. This value is an order of magnitude smaller than $V_{\text {SIA }} / V_{41}$ measured in GaAs ring samples with the strongest Rashba splitting. ${ }^{15}$ Hence, as a first approximation, it is admissible to neglect BIA spin splitting when discussing this experiment.

Formally, the BIA terms do not commute with $\hat{M}_{z}$, and the Ansatz given in Eq. (3) will not eliminate the $\varphi$ dependence from the BIA part of the ring Hamiltonian. In essence, previous eigenstates with quantum number $m$ are coupled via the BIA term to those with $m \pm 2$. A reduced-band model may be adequate to explore BIA effects in the lowest ring subband.

\section{CONCLUSIONS}

We have obtained numerically exact results for electronic subbands and spin-related geometric phases for holes in me- 
soscopic rings. Unlike previous models, we account fully for spin splitting and mixing arising in the quantum-confined valence band. For quasi-one-dimensional ring structures, a much stronger modulation of magnetoconductance oscillations (as a function of Fermi energy and/or SIA spin-splitting strength) is found as compared with simplified (purely $\mathrm{HH}$ ) models. This effect arises due to HH-LH mixing induced by the in-plane ring confinement, and the magnitude of the enhanced dependence is quantified by the parameter $\lambda_{d}$, which is related to the ratio of quantum-well width and in-plane ring width.

We have applied our model to discuss a recent experiment where an anomalously strong modulation of AharonovBohm oscillations was observed. A sizable enhancement of magnetoconductance-oscillation modulations is obtained within our (on some level still idealized) model, but its magnitude is smaller than the observed value. A more realistic modeling of the ring structure may be needed to reach full agreement. We also ascertained the effect of BIA spin splitting. The parameter quantifying its importance is the ratio of a length scale $\ell_{\text {BIA }}(=4.83 \AA$ in GaAs) and the quantum-well width, which was negligible compared to the strength of SIA splitting present in the experiment under consideration. Our theory, possibly with further refinement, should be useful for guiding efforts ${ }^{7,8}$ aimed at realizing novel electronic devices based on spin-dependent quantum interference.

\section{ACKNOWLEDGMENTS}

This work is supported by the Marsden Fund Council (Contract No. MAU0702) from Government funding, administered by the Royal Society of New Zealand.

\section{APPENDIX: DERIVATION OF EXPRESSIONS GIVEN FOR THE AHARONOV-ANANDAN PHASE AND THE MAGNETOCONDUCTANCE}

We assume a standard two-terminal transport geometry as shown, e.g., in Fig. 2 of Ref. 29. To keep the notation uncluttered, we consider the situation where only the lowest spin-split subband is relevant, but all formulas can be straighforwardly generalized to the multisubband case. Holes with energy $E$ are injected by an external lead at $\varphi=0$ in channel $s$ in a superposition of ring-state amplitudes: $\mid$ in $\rangle_{s}$ $=\xi_{s+}^{(\mathrm{in})} \chi_{s m_{+}}^{(s)}+\xi_{s-}^{(\mathrm{in})} \chi_{-s m_{-}}^{(s)}$. Here $\chi_{m}^{(s)}$ denotes the radial four-spinor wave function [see Eq. (3)] for an eigenstate from subband $s$, and the coefficients $\xi_{s \pm}^{(\text {in) }}$ depend on details of the coupling between ring and injecting lead. At a draining lead located diametrically opposite to the injecting one, holes from channel $s$ will enter with an amplitude

$$
\begin{aligned}
\mid \text { out }\rangle_{s} & =e^{i \pi\left(m_{+}-s \hat{J}_{z}\right)} \xi_{s+}^{(\mathrm{in})} \chi_{s m_{+}}^{(s)}+e^{i \pi\left(m_{-}+s \hat{J}_{z}\right)} \xi_{s-}^{(\mathrm{in})} \chi_{-s m_{-}}^{(s)} \\
& \equiv \operatorname{si\mathcal {M}}\left(e^{i \pi m_{+}} \xi_{s+}^{(\mathrm{in})} \chi_{s m_{+}}^{(s)}-e^{i \pi m_{-}} \xi_{s-}^{(\mathrm{in})} \chi_{-s m_{-}}^{(s)}\right),
\end{aligned}
$$

with the matrix $\mathcal{M}=\operatorname{diag}\{1,-1,1,-1\}$. Thus the phase difference between forward- and backward-propagating amplitudes is found to be $s \Theta_{\mathrm{G}}$ with $\Theta_{\mathrm{G}}$ given by Eq. (5). We have used the freedom that phases are determined only modulointeger multiples of $2 \pi$ to adjust $\Theta_{\mathrm{G}}$ such that it vanishes in the limit where $V_{\text {SIA }}=0$ and HH-LH mixing is neglected.

When the ring is penetrated by a magnetic flux $\Phi$ and coupled to ideal leads, the probability for transmission of holes is obtained as ${ }^{29,37}$

$$
T=\left|\sum_{s}\left[e^{i \pi\left(m_{+}+s \Phi / \Phi_{0}\right)} \xi_{s+}^{\text {(in) }} \xi_{s+}^{\text {(out) }}-e^{i \pi\left(m_{-}-s \Phi / \Phi_{0}\right)} \xi_{s-}^{\text {(in })} \xi_{s-}^{\text {(out })}\right]\right|^{2},
$$

where the factors $\xi_{s \pm}^{\text {(out) }}$ depend on the coupling between states $s \mathcal{M} \chi_{s m_{+}}^{(s)}$ and the scattering state in the outgoing lead. The two-terminal ring conductance $G_{\mathrm{rg}}=\frac{e^{2}}{2 \pi \hbar} T$ is then given, in full generality, by

$$
\begin{aligned}
G_{\mathrm{rg}}= & G_{0}\left[1+\sum_{s} A_{s}^{(1)} \cos \left(2 \pi \frac{\Phi}{\Phi_{0}}+s \Theta_{\mathrm{G}}\right)\right. \\
& \left.+A^{(2)} \cos \Theta_{\mathrm{G}}+A^{(3)} \cos \left(2 \pi \frac{\Phi}{\Phi_{0}}\right)\right] .
\end{aligned}
$$

The familiar contributions proportional to $A_{s}^{(1)}$ arise from interference between counter-propagating amplitudes from the same channel and manifest the AA phase. Additional interference terms (those proportional to $A^{(2,3)}$ ) are possible because coupling to leads may induce a mixing conductance between the two channels. In practice, this happens when the Hilbert space spanned by scattering states in the leads does not fully contain the space spanned by ring eigenstates. Such a situation could occur, in principle, because of the sensitive dependence of hole states on quantum confinement in the leads. As such effects will be small in typical situations and also depend strongly on the particular realizations of ringlead couplings, we have not considered them further in the context of this work. For similar reasons, we assume that the leads couple symmetrically to states in the two subbands. Setting $A^{(2)}=A^{(3)}=0$ and $A_{+}^{(1)}=A_{-}^{(1)}=A / 2$ yields Eq. (6), after application of an addition theorem for cosine functions.

\footnotetext{
${ }^{1}$ Mesoscopic Electron Transport, edited by L. L. Sohn, L. P. Kouwenhoven, and G. Schön (Kluwer, Dordrecht, 1997).

${ }^{2}$ R. A. Webb, S. Washburn, C. P. Umbach, and R. B. Laibowitz, Phys. Rev. Lett. 54, 2696 (1985); G. Timp, A. M. Chang, J. E. Cunningham, T. Y. Chang, P. Mankiewich, R. Behringer, and R. E. Howard, ibid. 58, 2814 (1987).
}

${ }^{3}$ M. V. Berry, Proc. R. Soc. London, Ser. A 392, 45 (1984).

${ }^{4}$ Y. Aharonov and J. Anandan, Phys. Rev. Lett. 58, 1593 (1987).

${ }^{5}$ J. Anandan, Nature (London) 360, 307 (1992).

${ }^{6}$ D. Loss and P. M. Goldbart, Phys. Rev. B 45, 13544 (1992); A. Stern, Phys. Rev. Lett. 68, 1022 (1992); A. G. Aronov and Y. B. Lyanda-Geller, ibid. 70, 343 (1993). 
${ }^{7}$ J. Nitta, F. E. Meijer, and H. Takayanagi, Appl. Phys. Lett. 75, 695 (1999).

${ }^{8}$ P. Földi, B. Molnár, M. G. Benedict, and F. M. Peeters, Phys. Rev. B 71, 033309 (2005).

${ }^{9}$ A. F. Morpurgo, J. P. Heida, T. M. Klapwijk, B. J. van Wees, and G. Borghs, Phys. Rev. Lett. 80, 1050 (1998).

${ }^{10}$ T. Bergsten, T. Kobayashi, Y. Sekine, and J. Nitta, Phys. Rev. Lett. 97, 196803 (2006).

${ }^{11}$ J. B. Yau, E. P. De Poortere, and M. Shayegan, Phys. Rev. Lett. 88, 146801 (2002).

${ }^{12}$ M. J. Yang, C. H. Yang, and Y. B. Lyanda-Geller, Europhys. Lett. 66, 826 (2004).

${ }^{13}$ M. König, A. Tschetschetkin, E. M. Hankiewicz, J. Sinova, V. Hock, V. Daumer, M. Schäfer, C. R. Becker, H. Buhmann, and L. W. Molenkamp, Phys. Rev. Lett. 96, 076804 (2006).

${ }^{14}$ B. Grbić, R. Leturcq, T. Ihn, K. Ensslin, D. Reuter, and A. D. Wieck, Phys. Rev. Lett. 99, 176803 (2007).

${ }^{15}$ B. Habib, E. Tutuc, and M. Shayegan, Appl. Phys. Lett. 90, 152104 (2007).

${ }^{16}$ N. Kang, E. Abe, Y. Hashimoto, Y. Iye, and S. Katsumoto, J. Phys. Soc. Jpn. 76, 083704 (2007).

${ }^{17}$ R. Winkler, Spin-Orbit Coupling Effects in Two-Dimensional Electron and Hole Systems (Springer, Berlin, 2003).

${ }^{18}$ D. Frustaglia and K. Richter, Phys. Rev. B 69, 235310 (2004).

${ }^{19}$ B. Molnár, F. M. Peeters, and P. Vasilopoulos, Phys. Rev. B 69, 155335 (2004).

${ }^{20}$ S. Souma and B. K. Nikolic, Phys. Rev. B 70, 195346 (2004).

${ }^{21}$ X. F. Wang and P. Vasilopoulos, Phys. Rev. B 72, 165336 (2005).

${ }^{22}$ Y. Tserkovnyak and A. Brataas, Phys. Rev. B 76, 155326 (2007).

${ }^{23}$ M. Wang and K. Chang, Phys. Rev. B 77, 125330 (2008).

${ }^{24}$ A. A. Kovalev, M. F. Borunda, T. Jungwirth, L. W. Molenkamp, and J. Sinova, Phys. Rev. B 76, 125307 (2007).

${ }^{25}$ M. F. Borunda, X. Liu, A. A. Kovalev, X.-J. Liu, T. Jungwirth, and J. Sinova, Phys. Rev. B 78, 245315 (2008).

${ }^{26}$ D. Stepanenko, M. Lee, G. Burkard, and D. Loss, Phys. Rev. B
79, 235301 (2009).

${ }^{27}$ Spin-dependent geometric phases due to SIA are generally energy dependent in hole systems - a distinguishing feature not shared by conduction-band electrons.

${ }^{28}$ J. M. Luttinger, Phys. Rev. 102, 1030 (1956).

${ }^{29}$ M. Pletyukhov and U. Zülicke, Phys. Rev. B 77, 193304 (2008).

${ }^{30} \mathrm{~B}$. Fornberg, A Practical Guide to Pseudospectral Methods (Cambridge University Press, Cambridge, UK, 1996).

${ }^{31}$ J. A. C. Weideman and S. C. Reddy, ACM Trans. Math. Softw. 26, 465 (2000).

${ }^{32}$ Ideal leads are perfectly absorbing, ensuring that charge carriers are traversing the ring only once (Ref. 33). The scattering matrix for an electronic $\mathrm{T}$ junction needed for describing the connection between a ring and an ideal lead was given first, for the spin rotationally invariant case, by B. Shapiro, Phys. Rev. Lett. 50, 747 (1983).

${ }^{33}$ Y. Gefen, Y. Imry, and M. Ya. Azbel, Phys. Rev. Lett. 52, 129 (1984); M. Büttiker, Y. Imry, and M. Ya. Azbel, Phys. Rev. A 30, 1982 (1984).

${ }^{34}$ Y. Aharonov and D. Bohm, Phys. Rev. 115, 485 (1959).

${ }^{35}$ D. Grundler, Phys. Rev. Lett. 84, 6074 (2000).

${ }^{36}$ In principle, our theory applies to the $n$-type device of Ref. 13 because a band inversion in the heterostructure from which the ring was fabricated renders the conduction band to have valence-band-like properties. However, this structure appears to be quite close to the $2 \mathrm{D}$ limit so that HH-LH mixing may not be very important.

${ }^{37}$ In principle, all orbital magnetic-field effects and Zeeman splitting are accounted for by our expressions if the $m_{ \pm}$are obtained from ring subbands that have been calculated with all pertinent terms in the Hamiltonian included. For simplicity, we neglect Zeeman splitting and magnetic-field-related distortions of inplane bound-state wave functions in this work. Our results are directly applicable to rings with sufficiently large aspect ratio where magnetoconductance oscillations can already appear in the weak-field regime. 